\title{
STRUCTURE OF THE ANTIEIGENVECTORS OF A STRICTLY ACCRETIVE OPERATOR
}

\author{
K.C. DAS, M.DAS GUPTA, K. PAUL \\ Department of Mathematics \\ Jadavpur University \\ Calcutta 700 032, India
}

(Received June 10, 1996 and in revised form March 6, 1997)

\begin{abstract}
A necessary and sufficient condition that a vector $f$ is an antieigenvector of a strictly accretive operator $\mathbf{A}$ is obtained. The structure of antieigenvectors of selfadjoint and certain class of normal operators is also found in terms of eigenvectors. The Kantorovich inequality for selfadjoint operators and the Davis's inequality for normal operators are then easily deduced. A sort of uniqueness is also established for the values of $\operatorname{Re}(A f, f)$ and $\|A f\|$ if the first antieigenvalue, which is equal to $\min \operatorname{Re}(\mathrm{Af}, \mathrm{f}) /(\|\mathrm{Af}\|\|f\|)$ is attained at the unit vector $\mathrm{f}$.
\end{abstract}

KEY WORDS AND PIRASES. Antieigenvectors, . Accretive Operator.

1991 AMS SUBJECT CLASSIFICATION CODES: Primary 47B44; 47A63 Secondary 47B15.

\section{INTRODUCTION}

Let $\mathrm{H}$ be a complex Hilbert space with inner product $($,$) , A a strictly accretive operator$ on $H$ and $A^{*}$ its adjoint. Let $\phi_{A}(f)=(A f, f) /\left(\|A f|\| f|), f \neq 0\right.$ and $\mu_{1}(A)=\min \left(\operatorname{Re} \phi_{A}(f)\right.$ : $f \in H, f \neq 0\}$.

Krein [1] and Gustafson [2] have studied $\mu_{1}(A)$ and indicated how the knowledge of $\mu_{1}(A)$ can be useful in the study of the spectrum of certain integral operators, initial value problems and some other areas. Geometrically $\mu_{1}(\mathrm{~A})$ represents the (real) cosine of the largest (real) angle through which an arbitary non-zero vector $f$ can be rotated by action of $A$. Gustafson calls it the first antieigenvalue of $\mathrm{A}$ and $\mathrm{f}$ the corresponding antieigenvector (See Remark 4.3, however, at the end of Section 4). The exact value of $\mu_{1}(\mathrm{~A})$ can be found in the case of a selfadjoint $A$. using the Kantorovich inequality $[2,3,4]$.

For a class of normal operators, Davis [3] obtained an inequality from which $\mu_{1}(A)$ can be found. He made use of the concept of a shell of a Hilbert space operator [5,6] to arrive at the results. Mirman [7] gave a method of estimation of $\mu_{k}(A)$, the higher antieigenvalues of $A$, which is defined by Gustafson as follows :

$$
\mu_{n}(A)=\min \left\{\operatorname{Re}(A f, f) /(\|A f\|\|f\|): f \neq 0, f \perp f^{(1)}, f^{(2)} \ldots . ., f^{(n-1)}\right\},
$$

where $f^{(k)}$ is the $k^{\text {th }}$ antieigenvector.

In the present note we put $\mu_{1}(\mathrm{~A})$ in a greater and clearer perspective by studying the 
structure of the vectors that make $\operatorname{Re}(A f, f)$ /(IAflllfil), $f \neq 0$, stationary. (For definition see Section 2). We call them stationary vectors. Clearly every antieigenvector is a stationary vector. We show that for selfadjoint and normal operators a suitable combination of two distinct eigenvectors yields. a stationary vector while the converse is true for all selfadjoint and a certain class of normal operators. Kantorovich inequality and Davis's inequality all follow as immediate consequences. If the first antieigenvalue. namely $\min \operatorname{Re} \phi_{A}(f)=\min \operatorname{Re}(A f, f) /(\|A f\|\|f\|), f \neq 0$ is attained for a unit vector $f$ then it is shown that the values of $\operatorname{Re}(A f . f)$ and $\| A f l$ are unique. An equation characterizing vectors for which $\left|\phi_{A}(f)\right|$ is stationary, is also given. A recursive variational scheme is used to define higher antieigenvalues.

\section{STATIONARY VALUES OF Re $\phi_{A}(f)$}

let $A$ be a strictly accretive operator on $H_{\text {and }}$ Let $B=\left(A+A^{*}\right) / 2$ and $C=A^{*} A$. Both the operators $B$ and $C$ are obviously positive definite. $\operatorname{Re} \phi_{A}(f)$ represents the cosine of the real angle of rotation of the vector $f$ under the action of $A$. Re $\phi_{A}(f)$ is said to have a stationary value at a vector $f(\neq 0)$ if the function $w_{B}(t)$ of a real variable $t$. defined by

$$
w_{g}(t)=\frac{(B f+t B g, f+t g)^{2}}{(C f+t C g, f+t g)(f+t g, f+t g)}
$$

has a stationary value at $\mathrm{t}=0$ for any arbitrary but fixed vector $\mathrm{g}$. In other words we must have $w_{g}^{\prime}(\eta)=0$ for all $g$. For $\|f\|=1$, set $(B f, f)=b$ and $(C f, f)=c^{2}$. With these notations. we see that $\operatorname{Re} \phi_{\Lambda}(f)$ is stationary at $f$ iff

$$
2 c^{2} \operatorname{Re}(B f, g)-b \operatorname{Re}(C f, g)-b c^{2} \operatorname{Re}(f, g)=0 .
$$

Since $g$ is arbitrary, we have the following theorem.

THEOREM 2.1. Let $\phi_{A}(f), b$ and $c^{2}$ be defined as above. A unit vector $f$ is a stationary vector of $\operatorname{Re} \phi_{A}(f)$ iff

$$
c^{2}\left(A+A^{*}\right) f-b A^{*} A f-b c^{2} f=0
$$

Equation (2.3) obviously characterizes the vectors for which $\operatorname{Re} \phi_{A}(f)$ is stationary, in particular. a minimum or a maximum.

COROLLARY 2.1. If for a stationary vector $f, A f=A^{*} f$ then $f$ is a linear combination of two eigenvectors $A f-c^{2} / b f \pm c / b \sqrt{c^{2}-b^{2}}$ f of $A^{*}$ with corresponding eigenvalues $c^{2} / b \pm$ $c / b \sqrt{c^{2}-b^{2}}$.

If in addition $A$ is normal then $f$ is a linear combination of two eigenvectors $A f-c^{2} / b f$ $\pm c / b \sqrt{c^{2}-b^{2}} f$ of $A$ with corresponding eigenvalues $c^{2} b \pm c / b \sqrt{c^{2}-b^{2}}$.

\section{STATIONARY VALUES OF $\Phi_{A}(f)$ FOR SELFADJOINT OPERATORS}

For a selfadjoint operator $A$ we can obtain the structure of the stationary vectors of Re申 $(f)$ (which is obviously equal to $\phi_{A}(f)$ in this case), in particular, of the antieigenvectors in terms of the eigenvectors. It will follow that $\mu_{1}(A)=2 \sqrt{\mathrm{mM}}(\mathrm{m}+\mathrm{M})^{-1}$, where as usual $\mathrm{m} . \mathrm{M}$ are the least and the greatest eigenvalues of $A$ respectively. This is the Kantorovich inequality (see [3] and $[4])$. We now state

THEOREM 3.1. A unit vector $f$ is a stationary vector of a selfadjoint operator $A$ iff there exists two eigenvectors whose appropriate linear combination (in the sense given below) yields $f$.

PROOF. If $\mathrm{f}$ is a stationary vector, in particular an antieigenvector then it satisfies (2.3). 
As $A$ is selfadjoint (2.3) reduces to

$\|A f\|^{2} 2 A f-(A f, f) A^{2} f-(A f . f)\|A f\|^{2} f=0$ which implies $A^{2} f-\left\|A f l / \phi_{A}(f) A f \pm\right\| h \| V \phi_{A}(f)$ Af $=\| A f l / \phi_{\Lambda}(f)$ Af $-\|A f\|^{2} f \pm\|h\| V \phi_{A}(f)$ Af where $h=A f-\lambda f$, with $(f, h)=0$ and $\lambda=(A f, f)$.

that is $\operatorname{A}\left[\operatorname{Af}-\|A f\| \phi_{A}(f) f \pm\|h\| \phi_{A}(f) f\right]=(\|A f\| \pm\|h\|) / \phi_{A}(f)\left[A f-\|A f\| / \phi_{A}(f) f \pm\|h\| V \phi_{A}(f) f\right]$

Let $\mu_{1}=(\|A f\|+\|h\|) / \phi_{A}(f), \mu_{2}=(\|A f\|-\|h\|) / \phi_{A}(f)$

$g_{1}=$ Af $-\mu_{2} f, g_{2}=A f-\mu_{1} f$, then $A g_{1}=\mu_{1} g_{1}, A g_{2}=\mu_{2} g_{2}$ and $f=1 /\left(\mu_{1}-\mu_{2}\right)\left(g_{1}-g_{2}\right)$.

So that $f$ is a linear combination of two eigenvectors.

Conversely, let $f=\alpha_{i} e_{i}+\alpha_{j} e_{j}$, with $\left|\alpha_{i}\right|^{2}=\frac{\lambda_{j}}{\lambda_{i}+\lambda_{j}}$ and $\left|\alpha_{j}\right|^{2}=\frac{\lambda_{i}}{\lambda_{i}+\lambda_{j}}$.

where $e_{i}$ and $e_{j}$ are any two eigenvectors of $A$ corresponding to eigenvalues $\lambda_{1}$ and $\lambda_{j}$.

So $b=(A f, f)=\lambda_{i}\left|\alpha_{i}\right|^{2}+\lambda_{j}\left|\alpha_{j}\right|^{2}=\frac{2 \lambda_{i} \lambda_{j}}{\lambda_{i}+\lambda_{j}}$ and $\dot{c}^{2}=\lambda_{i}{ }^{2}\left|\alpha_{1}\right|^{2}+\lambda_{j}{ }^{2}\left|\alpha_{j}\right|^{2}$. With these values we can (2.3) see that the equation (2.3) is satisfied by $f$. This completes the proof.

The Kantorovich inequality (see [2], [3] and [4]) now follows as a corollary.

COROLLARY 3.1. If $A$ is selfadjoint and $\mu_{1}(A)=\min \{(A f, f) /(\|A f\| \| t 11): f \in H . f \neq 0\}$ then $\mu_{1}(A)=2 \sqrt{m M} /(m+M)$.

PROOF. One need only to observe that $\mu_{1}(\mathrm{~A})$ is stationary value of $\phi_{\mathrm{A}}(\mathrm{f})$ and $2 \sqrt{\lambda_{i} \lambda_{j}} /\left(\lambda_{i}+\lambda_{j}\right)=2 /\left(\sqrt{\lambda_{i}} / \sqrt{\lambda_{j}}+\sqrt{\lambda_{j}} / \sqrt{\lambda_{i}}\right)$ assumes the least valuc for greatest $\lambda_{i} / \lambda_{j}$. which in this case is $\mathrm{M} / \mathrm{m}$.

\section{STATIONARY VALUES OF Re $\phi_{A}(f)$ FOR A NORMAL OPERATOR}

Before we discuss the structure of the stationary vectors in the normal case, we give a few examples to show that the situation in this case can be very different. In these examples $e_{k}$ ' $s$ are eigenvectors of $A$ corresponding to eigenvalues $\lambda_{k}$ 's, the values of which are clear from the context.

EXAMPLE 4.1. Let $A$ be normal with $A f=\Sigma(1+i k)\left(f, e_{k}\right) e_{k}, i=\sqrt{-1}$, $\|f\|=1$. So $b=$ $\Sigma\left|\left(f, e_{k}\right)\right|^{2}=1, c^{2}=\Sigma\left(1+k^{2}\right)|(f, e k)|^{2}$. Hence $b^{2} / c^{2}=1 /\left[\Sigma\left(1+k^{2}\right)\left|\left(f, e_{k}\right)\right|^{2}\right]$. Obviously, Re $\phi_{A}(f)$ attains a mininum at the eigenvector $e_{n}$ and $\mu_{1}(A)=1 /\left(1+n^{2}\right)$, where $n=\max \{k\}$.

EXAMPLE 4.2. Let $A f=(1+i)\left(f, e_{1}\right) e_{1}+(1-i)\left(f, e_{2}\right) e_{2}+(2+i)\left(f, e_{3}\right) e_{3}+(2-i)$ $\left(f, e_{4}\right) e_{4}$, where $i=\sqrt{-1},\|f\| l=1$. So $b=1+\left|\left(f, e_{3}\right)\right|^{2}+\left|\left(f, e_{4}\right)\right|^{2}$ and $c^{2}=2+3\left|\left(f, e_{3}\right)\right|^{2}+3\left|\left(f, e_{4}\right)\right|^{2}$. Hence $b^{2} / c^{2}$ will be a minimum for $\left|\left(f, e_{3}\right)\right|=\left|\left(f, e_{4}\right)\right|=0$, and then $\mu_{1}(A)=1 / \sqrt{2}$. Note that in this case $b=\operatorname{Re} \lambda_{i}$ and $c^{2}=\left|\lambda_{i}\right|^{2}$.

EXAMPLE 4.3. This is the most important example in this section. It shows that unlike the selfadjoint case, a linear combination of more than two eigenvectors corresponding to dinstinct eigenvalues may exist for the attainment of the minimum of $\operatorname{Re} \phi_{A}(f)$. Let us consider the normal operator $A$ such that

Af $=(1+i)\left(f, e_{1}\right) e_{1}+1 / 2(1+i)\left(f, e_{2}\right) e_{2}+(2+i)\left(f, e_{3}\right) e_{3}$ with $\left|\left(f, e_{1}\right)\right|^{2}+\left.\left|\left(f, e_{2}\right) R^{2}+\right|\left(f, e_{3}\right)\right|^{2}=1$, $\mathrm{i}=\sqrt{-1}$.

$$
\begin{aligned}
& \text { Clearly } b=\operatorname{Re}(A f, f)=\left|\left(f, e_{1}\right)\right|^{2}+\frac{1}{2}\left|\left(f, e_{2}\right)\right|^{2}+2\left|\left(f, e_{3}\right)\right|^{2}=1+\left|\left(f, e_{3}\right)\right|^{2} \\
& -\frac{1}{2}\left|\left(f, e_{2}\right)\right|^{2} \\
& \text { and } c^{2}=2\left|\left(f, e_{1}\right)\right|^{2}+\frac{1}{2}\left|\left(f, e_{2}\right)\right|^{2}+5\left|\left(f, e_{3}\right)\right|^{2} \\
& =2+3\left|\left(f, e_{3}\right)\right|^{2}-\frac{3}{2}\left|\left(f, e_{2}\right)\right|^{2}
\end{aligned}
$$

Hence $b^{2} / c^{2}=(1+k)^{2} /(2+3 k)$, where $k=\left|\left(f, e_{3}\right)\right|^{2}-\frac{1}{2}\left|\left(f, e_{2}\right)\right|^{2}$. For a minimum or a maximum. we must have $k=-1$ or $-1 / 3$. The case $k=-1$ must be ruled out as in that case $\left|\left(f, e_{2}\right)\right|^{2}>1$. 
For $k=-1 / 3$, we have $\operatorname{Re} \phi_{A}(f)=2 / 3$. Let $\left|\left(f, e_{1}\right)\right|^{2}=1 / 12,\left|\left(f, e_{2}\right)\right|^{2}=10 / 12$ and $\mid\left(f, e_{3}\right)^{2}=$ $\frac{10}{12}$.

So that unit vector $f=\frac{1}{\sqrt{12}} e_{1}+\frac{\sqrt{10}}{\sqrt{12}} e_{2}+\frac{1}{\sqrt{12}} e_{3}$ will be the first antieigenvector of A. However it is possible to have a combination of only two eigenvectors corresponding to two eigenvalues for which the minimum in question is attained. Set $\left|\left(f, e_{3}\right)\right|=0,\left|\left(f, e_{2}\right)\right|^{2}=\frac{2}{3}$ and $\left|\left(f, e_{1}\right)\right|^{2}=$ $\frac{1}{3}$. Clearly $f=\frac{1}{\sqrt{3}} e_{1}+\frac{\sqrt{2}}{\sqrt{3}} e_{2}$ will be a required vector.

THEOREM 4.1 : Let $A$ be normal with a complete orthonormal set of eigenvectors $e_{i}$ and corresponding eigenvalues $\lambda_{i}$ such that for any unit vector $f \in H$, Af $=\Sigma \lambda_{i}\left(f, e_{i}\right) e_{i}$.

If $\operatorname{Re} \phi_{A}(f)$ is stationary at $f$, and $f$ is not an eigenvector of $A$, then either $f$ is a linear combination of two eigenvectors corresponding to two conjugate eigenvalues or there exists a linear combination of two eigenvectors corresponding to two eigenvalues with unequal real parts and moduli, say $g$, such that $\operatorname{Re} \phi_{A}(g)=\operatorname{Re} \phi_{A}(f)$ and $\operatorname{Re} \phi_{A}$ is stationary at $g$.

Further, we have the relation

$$
\operatorname{Re}^{2} \phi_{\Lambda}(f)=\frac{4 \lambda \mu(\mu \cos \theta-\lambda \cos \phi)(\mu \cos \phi-\lambda \cos \theta)}{\left(\lambda^{2}-\mu^{2}\right)^{2}}
$$

if $\lambda e^{\mathrm{i} \theta}$ and $\mu \mathrm{e}^{\mathrm{i} \phi}$ are the distinct eigenvalues referred to above and are such that $\lambda \neq \mu$ and $\lambda \cos \theta \neq \mu \cos \phi$.

PROOF. If $\operatorname{Re} \phi_{A}(f)$ is stationary at $f$, we must have

$$
c^{2}\left(A+A^{*}\right) f-b A^{*} A f-b c^{2} f=0
$$

Substitute $f=\Sigma\left(f, e_{1}\right) e_{i}$, Af $=\Sigma \lambda_{i}\left(f, e_{i}\right) e_{i}$ and $A^{*} f=\Sigma \bar{\lambda}_{i}\left(f, e_{1}\right) e_{i}$, in (4.1) to get

$$
2 c^{2} \operatorname{Re} \lambda_{i}-b\left|\lambda_{i}\right|^{2}-b c^{2}=0
$$

if $\left(f, e_{i}\right) \neq 0$

As in example 4.1, (4.2) may be satisfied if $A f=\lambda f, b=\operatorname{Re} \lambda, c^{2}=|\lambda|^{2}$. If $f$ is not an eigenvector then (4.2) may be satisfied by only one pair $\left(\operatorname{Re} \lambda_{i},\left|\lambda_{i}\right|^{2}\right)$ in which case, since $f$ is not an eigenvector, $\left(f, e_{i}\right) \neq 0$ for at least two $e_{i}$ 's corresponding to two distinct $\lambda_{i}$ 's. So, the only way this can happen is that $A f=\lambda\left(f, e_{1}\right) e_{1}+\bar{\lambda}\left(f, e_{2}\right) e_{2}$ where the renumbering of $e$ 's is obvious. Here $b=\operatorname{Re} \lambda, c^{2}=|\lambda|^{2}$. This is the case in example 4.2.

If (4.2) is satisfied by more than one pair $\left(\operatorname{Re} \lambda_{i},\left|\lambda_{i}\right|^{2}\right)$ then as in example 4.3 , the stationary vector $f$ may be a linear combination of more than two eigenvectors corresponding to distinct eigenvalues. We shall show, however, that there always exist a suitable linear combination $g$ of two eigenvectors corresponding to two eigenvalues of distinct moduli and real parts, such that $\operatorname{Re} \phi_{A}(g)=\operatorname{Re} \phi_{A}(f)$ and $\operatorname{Re} \phi_{A}$ is stationary at $g$. Let $\lambda_{1}=\lambda e^{1 \theta}$ and $\lambda_{j}=\mu e^{i \phi}, \lambda \neq \mu$ and $\lambda \cos \theta \neq \mu \cos \phi$ satisfy (4.2). We will find $\alpha_{i}$ and $\alpha_{j}$ such that $g=\alpha_{i} e_{i}+\alpha_{j} e_{j},\left|\alpha_{i}\right|^{2}+\left|\alpha_{j}\right|^{2}$ $=1, b=\operatorname{Re}(\mathrm{Af}, \mathrm{f})=\operatorname{Re}(\mathrm{Ag}, \mathrm{g}), \mathrm{c}^{2}=(\mathrm{Af}, \mathrm{Af})=(\mathrm{Ag}, \mathrm{Ag})$ and $(4.1)$ is satisfied for $\mathrm{g}$. Now $\mathrm{b}=$ $\left|\alpha_{i}\right|^{2} \lambda \cos \theta+\left|\alpha_{j}\right|^{2} \mu \cos \phi$ and $c^{2}=\left|\alpha_{i}\right|^{2} \lambda^{2}+\left|\alpha_{j}\right|^{2} \mu^{2}$ :

Since $\left|\alpha_{1}\right|^{2}+\left|\alpha_{1}\right|^{2}=1$, we get

$$
\left|\alpha_{i}\right|^{2}=\frac{b-\mu \cos \phi}{\lambda \cos \theta-\mu \cos \phi} \text { and also }\left|\alpha_{i}\right|^{2}=\frac{c^{2}-\mu^{2}}{\lambda^{2}-\mu^{2}} .
$$


But since $2 c^{2} \lambda \cos \theta-b \lambda^{2}-b c^{2}=0$ and $2 c^{2} \mu \cos \phi-b \mu^{2}-b c^{2}=0$, the value of $\left|\alpha_{1}\right|^{2}$ is the same. Hence a $g$ is obtained. A simple calculation further shows that

$$
\frac{b^{2}}{c^{2}}=\frac{4 \lambda \mu(\mu \cos \theta-\lambda \cos \phi)(\mu \cos \phi-\lambda \cos \theta)}{\left(\lambda^{2}-\mu^{2}\right)^{2}}
$$

and that completes the proof.

REMARK 4.1. It may be noted that (4.3) is equivalent to the inequality of Davis [3] when $b^{2} / c^{2}$ is a minimum of $\operatorname{Re} \phi_{A}(f)$ without the restrictive conditions (in part (ii) of his Theorem).

REMARK 4.2. The points of the line $2 c^{2} X-b Y-b c^{2}=0$ lying in the region $Y \geq X^{2}$ are such that

$$
\frac{c^{2}-c^{2}\left(1-(b / c)^{2}\right)^{1 / 2}}{b} \leq x \leq \frac{c^{2}+c^{2}\left(1-(b / c)^{2}\right)^{1 / 2}}{b}
$$

Hence all eigenvalues $\lambda$ with $\operatorname{Re} \lambda$ lying between the above limits and $\operatorname{Im} \lambda$ determined from (4.2) may contribute corresponding eigenvectors to yield a stationary vector of $\operatorname{Re} \phi_{A}(f)$.

REMARK 4.3. Since min $\operatorname{Re} \phi_{A}(f)$ may be attained even for an eigenvector as in example 4.1, Gustafson's choice to call it an antieigenvalue does not seem to be a happy one.

\section{FURTHER PROPERTIES AT $\min \phi_{A}$ (f) AND VARIATIONAL CHARACTERIZATION}

Consideration of the second derivative of $w_{B}(t)$, as defined in equation (2.1), shows that $w_{B}{ }^{\prime \prime}(0) \geq 0$ is a necessary condition for a unit vector $f$ to be the first antieigenvector. A rather lengthy calculation shows that then

$$
4 c^{2} \operatorname{Re}^{2}(\mathrm{Bf}-\mathrm{bf}, \mathrm{g})+2 b c^{2}(\mathrm{Bg}, \mathrm{g})-\mathrm{b}^{2}(\mathrm{Cg}, \mathrm{g})-\mathrm{b}^{2} \mathrm{c}^{2}(\mathrm{~g}, \mathrm{~g}) \geq 0
$$

for arbitary $g \in \mathrm{H}$, and $b, c$ defined as before. The quantities $b$ and $c$ are unique in the sense of the following theorem.

THEROM 5.1. If the $\min \phi_{A}(f)$ is attained for a unit vector $f$ with $b=(B f, f)$ and $c^{2}=$ $(C f, f)$, then there cannot exist unit vectors $g$ such that $(B g, g)=k b$ and $(C g, g)=k^{2} c^{2}, k \neq 1$.

PROOF. If such a $g$ exists then without loss of generality we can have $\operatorname{Re}(\mathrm{Bf}-\mathrm{bf}, \mathrm{g})=0$. Hence we get from (5.1), $2 \mathrm{~kb}^{2} \mathrm{c}^{2}-\mathrm{k}^{2} \mathrm{~b}^{2} \mathrm{c}^{2}-\mathrm{b}^{2} \mathrm{c}^{2} \geq 0$ or $-\mathrm{b}^{2} \mathrm{c}^{2}(\mathrm{k}-1)^{2} \geq 0$ which is absurd if $\mathbf{k} \neq 1$.

Higher antieigenvalues may be defined in a recursive variational manner as follows :

$$
\begin{aligned}
\mu_{1}(A)= & \min \operatorname{Re} \phi_{A}(f)=\operatorname{Re} \phi_{A}\left(f_{1}\right) \\
& f \in H \\
\mu_{n+1}(A) & =\min \operatorname{Re} \phi_{A}(f) \\
& f \perp f_{1} \ldots \ldots f_{n} \\
& f \perp B f_{1} \ldots \ldots . . B f_{n}
\end{aligned}
$$

Simplification in the selfadjoint case is obvious where eigenvectors get eliminated pairwise.

Gustafson [2] defines imaginary and total antieigenvalues by $\min \operatorname{Im} \phi_{A}(f)$ and $\min \left|\phi_{A}(f)\right|$ respectively. While the equation characterizing imaginary antieigenvectors will be similar to our equation (2.3), that for total antieigenvectors will be slightly different. If $f$ is the unit vector for which $\min \left|\phi_{A}(f)\right|$ is attained, then we must have

$$
\begin{gathered}
2 c^{2}\left(b_{x} A_{x}-b_{Y} A_{Y}\right) f-|b|^{2} C f-|b|^{2} c^{2} f=0 \\
\text { where } A_{x}=\left(A+A^{*}\right) / 2, A_{Y}=\left(A-A^{*}\right) / 2 i, C=A^{*} A \\
b=(A f, f), b_{x}=\operatorname{Re}(A f, f), b_{Y}=\operatorname{Im}(A f, f) \text { and } c^{2}=(C f, f) .
\end{gathered}
$$




\section{REFERENCES}

1. KREIN, M. G., Angular localization of the spectrum of a multiplicative integral in Hilbertspace, Functional Anal and its Appl 3 (1969), 73-74.

2. GUSTAFSON, K., Antieigenvalue inequalities in operator theory in Inequalities; O.Shisha ed., Vol.III, Academic, New York, 1972, pp. 115-119.

3. DAVIS, CH., Extending the Kantorovich inequality to normal matrices, Linear Alg. and its Appl 31 (1980), 173-177.

4. MARCUS, M. and MINC, H., A Survey of Matrix Theory and Matrix Inequalities, Allyn and Bacon, New York, 1964.

5. DAVIS, CH., The shell of a Hilbert-space operator, Acta Sci. Math. (Szeged) 29 (1968), 69-86.

6. DAVIS, CH., The shell of a Hilbert-space operator-II, Acta Sci.Math. (Szeged) 31 (1970), 301-318.

7. MIRMAN, B., Antieigenvalues : Method of Estimation and Calculation, Linear Alg. and its Appl. 49 (1983), 247-255. 


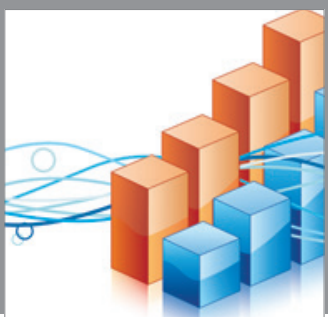

Advances in

Operations Research

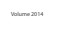

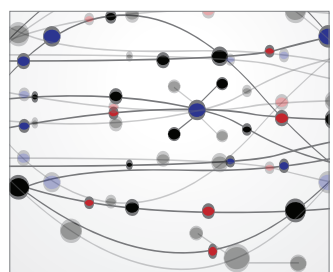

\section{The Scientific} World Journal
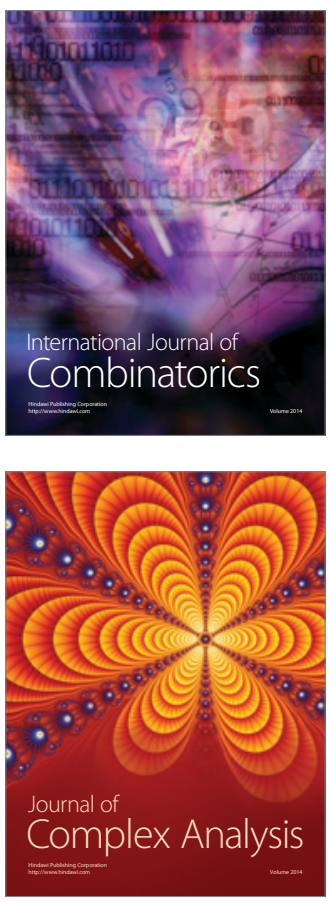

International Journal of

Mathematics and

Mathematical

Sciences
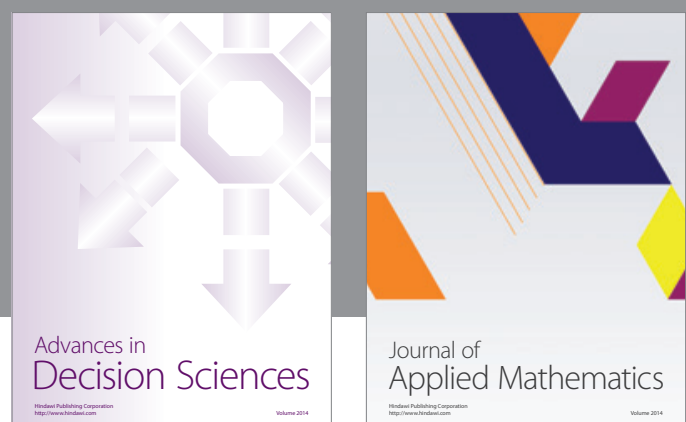

Journal of

Applied Mathematics
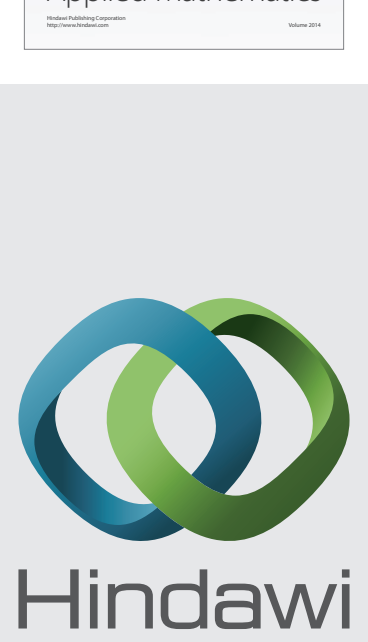

Submit your manuscripts at http://www.hindawi.com
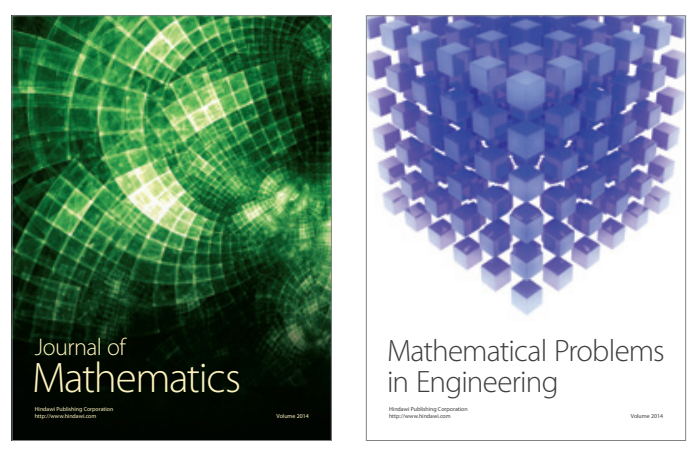

Mathematical Problems in Engineering
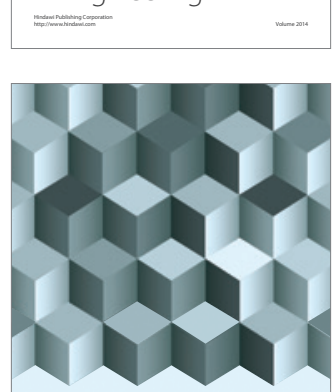

Journal of

Function Spaces
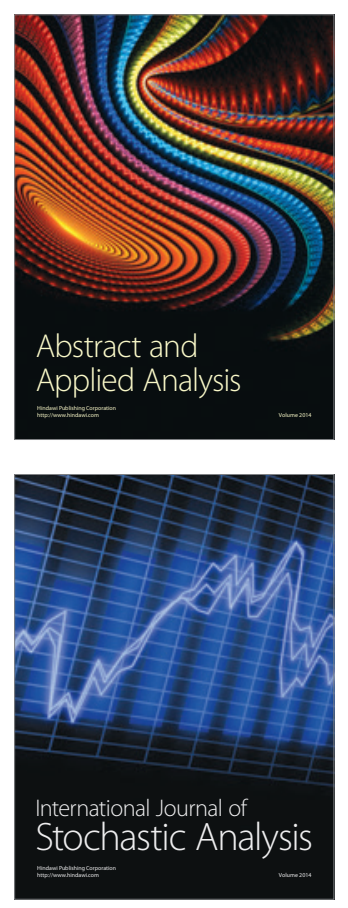

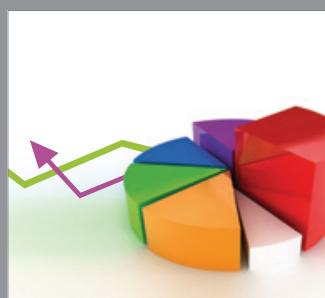

ournal of

Probability and Statistics

Promensencen
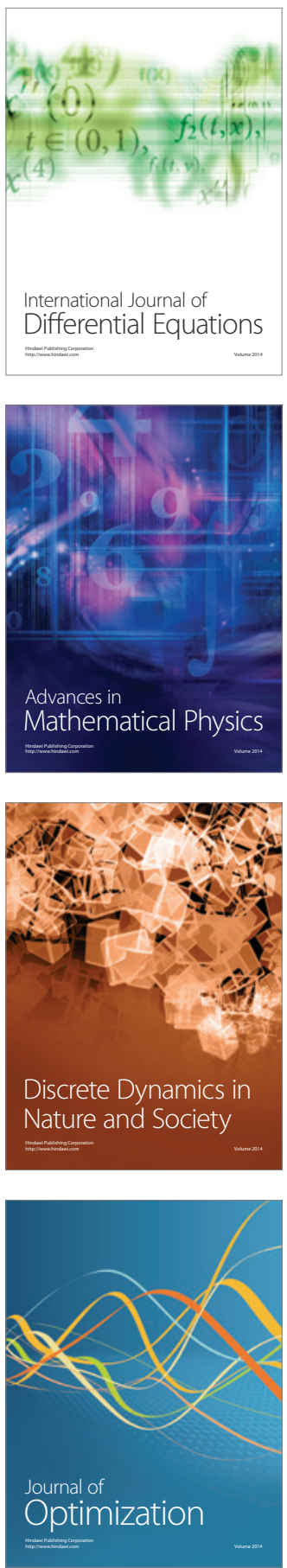